\title{
Isolation, Screening, and Identification of Novel Isolates of Actinomycetes from India for Antimicrobial Applications
}

\author{
Vineeta Singh ${ }^{1,2 *}$, Shafiul Haque ${ }^{3,4}$, Harshita Singh ${ }^{1}$, Jyoti Verma ${ }^{1}$, Kumari Vibha ${ }^{1}$, \\ Rajbir Singh ${ }^{5}$, Arshad Jawed ${ }^{4,6}$ and C. K. M. Tripathi ${ }^{4,7}$ \\ ${ }^{1}$ Microbiology Division, Council of Scientific and Industrial Research-Central Drug Research Institute, Lucknow, India, \\ ${ }^{2}$ Department of Biotechnology, Institute of Engineering and Technology, Lucknow, India, ${ }^{3}$ Department of Biosciences, Jamia \\ Millia Islamia (A Central University), New Delhi, India, ${ }^{4}$ Research and Scientific Studies Unit, College of Nursing and Allied \\ Health Sciences, Jazan University, Jazan, Saudi Arabia, ${ }^{5}$ Fermentation Technology Division, Council of Scientific and \\ Industrial Research-Central Drug Research Institute, Lucknow, India, ${ }^{6}$ Department of Biotechnology, Himachal Pradesh \\ University, Shimla, India, ${ }^{7}$ Department of Biotechnology, Shri Ramswaroop Memorial University, Lucknow, India
}

\section{OPEN ACCESS}

Edited by:

Vijai Kumar Gupta,

National University of Ireland, Ireland

Reviewed by:

Alok Kumar Pandey,

International Centre for Genetic Engineering and Biotechnology, India

Praveen Chandra Verma,

Council of Scientific and Industrial Research-National Botanical Research Institute, India

*Correspondence: Vineeta Singh vscdri@gmail.com

Specialty section:

This article was submitted to Microbiotechnology, Ecotoxicology and Bioremediation,

a section of the journal

Frontiers in Microbiology

Received: 09 July 2016 Accepted: 15 November 2016 Published: 06 December 2016

Citation:

Singh $V$, Haque $S$, Singh $H$, Verma J,

Vibha $K$, Singh $R$, Jawed $A$ and

Tripathi CKM (2016) Isolation, Screening, and Identification of Novel Isolates of Actinomycetes from India

for Antimicrobial Applications.

Front. Microbiol. 7:1921.

doi: 10.3389/fmicb.2016.01921
The search for novel bioactive compounds from the natural environment has rapidly been gaining momentum with the increase in multi-drug resistant (MDR) pathogens. In the present study, the antimicrobial potential of novel actinomycetes has been evaluated by initial screening of six soil samples. Primary and secondary screening was performed against Bacillus subtilis, Staphylococcus aureus, Escherichia coli, Candida albicans, Candida tropicalis, Trichophyton rubrum, and other MDR bacterial and fungal test strains, thirteen active isolates were selected for further study. Microbial strains were identified on the basis of growth conditions and other biochemical characters. Five most active microbial strains were identified using 16S rRNA sequence homology and designated as Streptomyces xanthophaeus MTCC 11938, Streptomyces variabilis MTCC 12266, Streptomyces xanthochromogenes MTCC 11937, Streptomyces levis EU 124569, and Streptomyces sp. NCIM 5500. Four antibacterial and three antifungal compounds isolated from the above five isolates were purified and partially characterized using UV absorption and IR spectra. Two antibacterial metabolites, belong to chromone and peptide antibiotic, respectively. The antifungal compounds were found to be of non-polyene nature. In conclusion, we study the isolation of novel bacterial strains of actinomycetes for producing novel compounds having antibacterial and antifungal activities from the unexplored agro-ecological niches of India. Also, this study paves the way for further characterization of these isolates of Streptomyces sp. for their optimum utilization for antimicrobial purposes.

Keywords: antibiotic production, antimicrobial activity, Streptomyces sp., chromone antibiotics, non-polyenes

\section{INTRODUCTION}

The search for bioactive metabolites including novel antibiotic compounds from microbial sources for potential use in agricultural, pharmaceutical, and industrial applications has become more important due to the development of drug/multi-drug resistance in most of the pathogenic microbes. Researchers across the globe are aggressively searching for new, 
potent, sustainable, and broad-spectrum antimicrobial compounds from various sources including microbes (Berdy, 2005; Hayakawa, 2008; Praveen et al., 2008; Singh and Tripathi, 2011). In natural soil habitat, Streptomyces are usually a major proportion of the total actinomycetes population and recognized as prolific producers of useful bioactive compounds (Tanaka and Omura, 1990; Kekuda et al., 2014).

Traditional screening methods have led to the isolation of common microorganisms capable of producing metabolites, which have already been extensively studied and established (Okami and Hotta, 1988; Kurtböke et al., 1992). Among the current strategies of natural-product screening, improved methodologies for isolating the uncommon and less studied rare actinomycetes are required to avoid the repeated isolation of the strains that produce known bioactive metabolites, and to improve the quality of the screened natural products (Takahashi and Omura, 2003; Berdy, 2005; Singh et al., 2009).

Likewise, the traditional methods of species classification and the identification of the organism(s) are mainly based on morphological, physiological, biochemical, developmental, and nutritional characters, and it is not adequate, and warrants for the use of molecular level approaches for assigning accurate taxonomic classification. Hence, precise assignment of taxonomic status to the novel bioactive microbial isolates through existing predictive bioinformatics methods and tools are very essential and aid in chemical characterization of the active molecules.

India has a unique asset of biodiversity, which can be used as a treasure for the search of novel isolates. With the variation of type of soil, according to the geographical changes, soil provide very complex habitat to the microbes residing in it. Due to this intricate environment, the soil microbes play an important role in the isolation of novel drugs. Among soil microbes, the members of Streptomyces sp. of actinomycetes, have been widely exploited for the production of commercially important secondary metabolites and enzymes. In the present study, the soil samples from six different unexplored agro-ecological niches of India have been screened-out to isolate Streptomyces sp. possessing antibacterial and antifungal activities.

\section{MATERIALS AND METHODS}

\section{Collection of Soil Samples}

The soil samples were collected from six diverse habitats of India (Lucknow, Uttar Pradesh: $26.7^{\circ} \mathrm{N}, 80.9^{\circ} \mathrm{E}$; Badrinath, Uttarakhand: $30.7440^{\circ} \mathrm{N}, \quad 79.4930^{\circ} \mathrm{E}$; Delhi, New Delhi: $28.6100^{\circ} \mathrm{N}, 77.2300^{\circ} \mathrm{E}$; Bhatinda, Punjab: $30.2300^{\circ} \mathrm{N}, 74.9519^{\circ}$ E; Haryana: $28.04^{\circ} \mathrm{N} 76.11^{\circ} \mathrm{E}$; Thinmala range, Kerala: $8.5074^{\circ} \mathrm{N}$, $76.9730^{\circ} \mathrm{E}$ ) for the isolation of microbes. These habitats included rhizosphere of the plants, agricultural soil, hospital surroundings, river mud, and preserved areas of forest soils. The samples were collected from upto $20 \mathrm{~cm}$ depth after the removal of $\sim 3.0 \mathrm{~cm}$ of the soil from the surface. The soil samples were collected in polyethylene bags, sealed, and stored in a refrigerator. All chemicals, media, media components, and other reagents were purchased from Sigma-Aldrich (USA), Merck (USA), HiMedia Laboratories (India) etc.

\section{Pre-treatment of Soil Samples and Isolation of Cultures}

The soil samples collected from different geographical areas were pre-treated to eliminate the commonly found microbes using physico-chemical methods. For the physico-chemical treatment of the soil samples, one gram of each soil sample was suspended in $10 \mathrm{ml}$ of normal saline and distributed in aliquots. One aliquot of the soil sample was treated with heat for $1 \mathrm{~h}$ at $120^{\circ} \mathrm{C}$ and the other was treated with $1.5 \%$ phenol for $30 \mathrm{~min}$ at $30^{\circ} \mathrm{C}$ as described by Hayakawa et al. (1991). Afterwards, the physicochemically treated soil samples were vortexed and left for 30 min, there after soil samples were serially diluted and $100 \mu \mathrm{l}$ of each "dilution" was plated on nutrient agar (NA), actinomycetes isolation agar (AIA), yeast malt glucose agar (M6), antibiotic assay agar, starch casein agar, and Czapek Dox agar.

\section{Screening of Microbial Cultures}

Primary screening for evaluating the antimicrobial potential of the axenic cultures was performed by perpendicular streak method of Madigan et al. (1997) against the bacterial strains of Bacillus subtilis MTCC 441, Staphylococcus aureus MTCC 96, Escherichia coli MTCC 64, and Candida albicans MTCC 183. Isolates were screened for antagonism studies by inoculating a single streak of the pure producer organism in the middle of the assay media plate. The plates were incubated for 4 days at $28^{\circ} \mathrm{C}$ and subsequently seeded with "test" organism by a single streak at a $90^{\circ}$ angle to the streak of the "producer strain" and finally the plates were incubated for $1-2$ days at $28^{\circ} \mathrm{C}$. The microbial interactions were analyzed by determining the distance of inhibition measured in $\mathrm{mm}$.

Microbial strains showing "moderate" to "good" inhibition activity were selected for secondary screening, which was performed by agar well method (Wu, 1984), using $100 \mu \mathrm{l}$ of their fermented broth against B. subtilis MTCC 441, B. subtilis MTCC 121, B. pumilus MTCC 1607, S. aureus MTCC 902, S. aureus MTCC 96, E. coli MTCC 1304, Salmonella typhi MTCC 734, Pseudomonas aeruginosa MTCC 741, Proteus vulgaris MTCC 426, C. albicans MTCC 3017, C. albicans MTCC 183, C. tropicalis MTCC 184, Saccharomyces cerevisiae MTCC 170, Cryptococcus terreus MTCC 1716, Aspergillus niger MTCC 1344, Trichophyton rubrum MTCC 296, Penicillium chrysogenum MTCC 2725, and Beauveria bassiana MTCC 4564. All the experiments were performed in triplicate and the average values were considered for analysis.

\section{Characterization of Microbial Strain(s) from the Selected Cultures}

The cultural characteristics of the producer strains were studied according to the method of Shirling and Gottlieb (1966) based upon their intensity of growth, growth pattern, colony color along with the color of aerial and substrate mycelia, and the formation of soluble pigments on oat meal agar (ISP-3), inorganic salt starch agar (ISP-4), glycerol asparagine agar (ISP-5), peptone yeast extract iron agar (ISP6), and tyrosine agar (ISP-7). The strains were characterized by streaking the culture(s) on the 
above mentioned medium plates and observed after 7-10 days of incubation at $28^{\circ} \mathrm{C}$ for the given characteristics.

Physiological and biochemical tests were performed as described by Williams et al. (1989) and Bergey's manual (Holt et al., 1994), and results were observed after 10 days of incubation of plates at $28^{\circ} \mathrm{C}$. In addition, the strains were tested for nitrate reduction, tolerance to $\mathrm{NaCl}$, decomposition of citrate, tartrate, acetate and pyruvate, and $\mathrm{pH}$ and temperature tolerance. The enzymatic activity assays of urease, amylase, protease and catalase were performed as suggested by Hopwood and Wright (1973). Finally, on the basis of macroscopic, biochemical and physiological characteristics hierarchical cluster analysis was performed by using PASW Statistics (formerly SPSS Statistics) Version 18 software program and dendrogram was generated based on the average linkage between the groups.

\section{Metabolite Production}

Pure and active cultures of microbial strains selected from the secondary screening experiments were grown in X-medium $(\mathrm{g} / \mathrm{l}$ : Soybean meal, 10; $\mathrm{CaCO}_{3}, 3 ; \mathrm{MgSO}_{4} .7 \mathrm{H}_{2} \mathrm{O}, 0.5 ;\left(\mathrm{NH}_{4}\right)_{2} \mathrm{HPO}_{4}$, 0.5; NaCl, 3; $\mathrm{K}_{2} \mathrm{HPO}_{4}$, 1; glycerol, 15 ml; DW 1, pH 6.9-7.0), and incubated at $28^{\circ} \mathrm{C}$ for 3-5 days and cellular growth was confirmed by visible pellets, clumps, aggregates or turbidity in the culture broth. The culture broths were centrifuged separately and filtrates were used to evaluate the antimicrobial activity against the above mentioned test microorganisms. Antibiotic activities of the strains were compared with that of known commercially available erythromycin $\left(\mathrm{E}^{15}\right)$ and amphotericin $\mathrm{B}\left(\mathrm{AmB}^{100}\right)$.

\section{Extraction, Purification, and Partial Characterization of the Active Compounds}

Fermented culture was centrifuged at $10,000 \mathrm{rpm}$ for $20 \mathrm{~min}$ to separate the biomass. The active metabolite was recovered from the fermented broth using two phase solvent extraction system with organic solvent. Solvents containing the active compounds were concentrated under vacuum to get "dried crude." The obtained "crude" was treated with non-polar solvents like hexane or chloroform to separate the polar and nonpolar components. The active components were purified by adsorption chromatography using silica gel (pore size $60 \AA$, mesh size: $230-400$, particle size $40-63 \mu \mathrm{m}$ ) as a stationary phase and gel filtration chromatography using sephadex LH20. The eluted fractions were assayed for their bioactivity against B. subtilis ATCC 6633 and C. albicans ATCC 24433 by disc diffusion method ( $\mathrm{Wu}, 1984)$. The purity of the active fractions was further checked by high pressure liquid chromatography (HPLC) using reverse phase silica column (RP18). Finally, the UV spectra (Perkin Elmer Lambda-25 UV spectrophotometer) of various antibacterial and antifungal compounds were determined in methanol at 200-500 $\mathrm{nm}$ wave length.

\section{RESULTS}

\section{Screening of the Active Strains}

During the screening, thirty six actinomycetes were isolated from six different stressed agro-ecological niches of India.

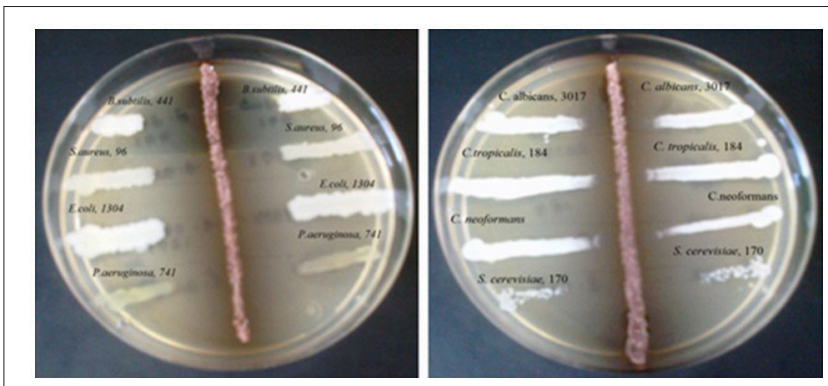

FIGURE 1 | Primary screening using perpendicular streak method for antibacterial and antifungal activity of the soil isolate (ZA25).

Microbial colonies showing distinct morphological characters were selected for the primary screening (Figure 1). Out of 36 total actinomycetes isolates, 15 showed moderate to strong antimicrobial activity against gram positive (B. subtilis MTCC6633, S. aureus MTCC 6538), gram negative (E. coli MTCC 1304) and fungal strain (C. albicans MTCC 1346). The active strains (actinomycetes) were further subjected to the secondary screening against some multi-drug resistant (MDR) bacterial as well as fungal test strains (Tables 1, 2). The screening results suggested that most of the isolates were active against gram positive bacteria in comparison with gram negative bacteria (Figures 2A,B). Out of 15 active isolates, 13 showed strong antimicrobial activity and were selected for detailed taxonomic, physiological, and biochemical studies.

\section{Characterization of the Strains}

The cultural characteristics, such as microbial growth along with its pattern and pigment formation were studied on International Streptomyces Project (ISP) media and their results are summarized in Table 3. All the selected actinomycetes isolates showed moderate to heavy growth on nitrate agar, urease agar and inorganic salt starch agar medium, which suggests their capability of breaking down the above complexes. Heavy growth and color change (from green to blue) of Simmons' Citrate Agar (SCA) medium provide the evidence of citrate utilizing ability of the actinomycetes strains YE21, YE22, and YE23. Likewise, melanin formation was demonstrated by the presence of brown to black patches on inorganic salt starch agar (ISP4) and YMG (yeast extract $4 \mathrm{~g}$, malt extract $10 \mathrm{~g}$, glucose $4 \mathrm{~g}$, agar $20 \mathrm{~g}$, water $1 \mathrm{~L})$ medium as a result of the growth of the active strains. This was observed in almost all the selected strains.

All the strains were capable of reducing nitrate salts but they demonstrated some differences against the decomposition of starch and urea. The production of amylase, urease and protease enzymes by the actinomycetes strains was endorsed by their growth on ISP4, urease agar and ISP3 mediums. Except the actinomycetes strains ZA25, ZA26, and YE22, the most favorable temperature range for the growth of strains was 27$37^{\circ} \mathrm{C}$. Interestingly, none of the selected strains were able to grow at $50^{\circ} \mathrm{C}$ or above. All the selected actinomycetes strains possessed the ability to tolerate $3 \% \mathrm{NaCl}$ in the medium with the exception of strains ZE18 and ZA25. 

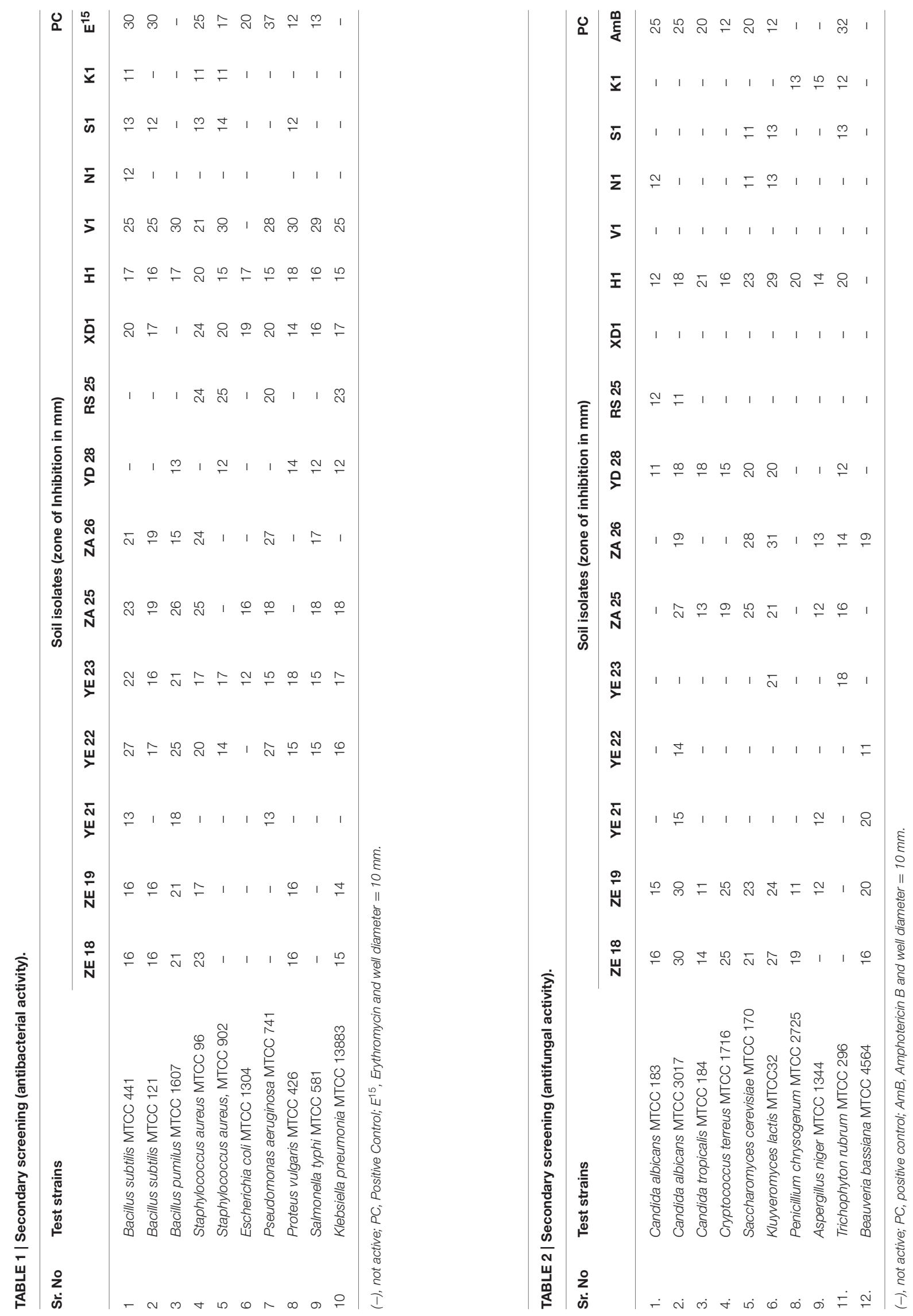


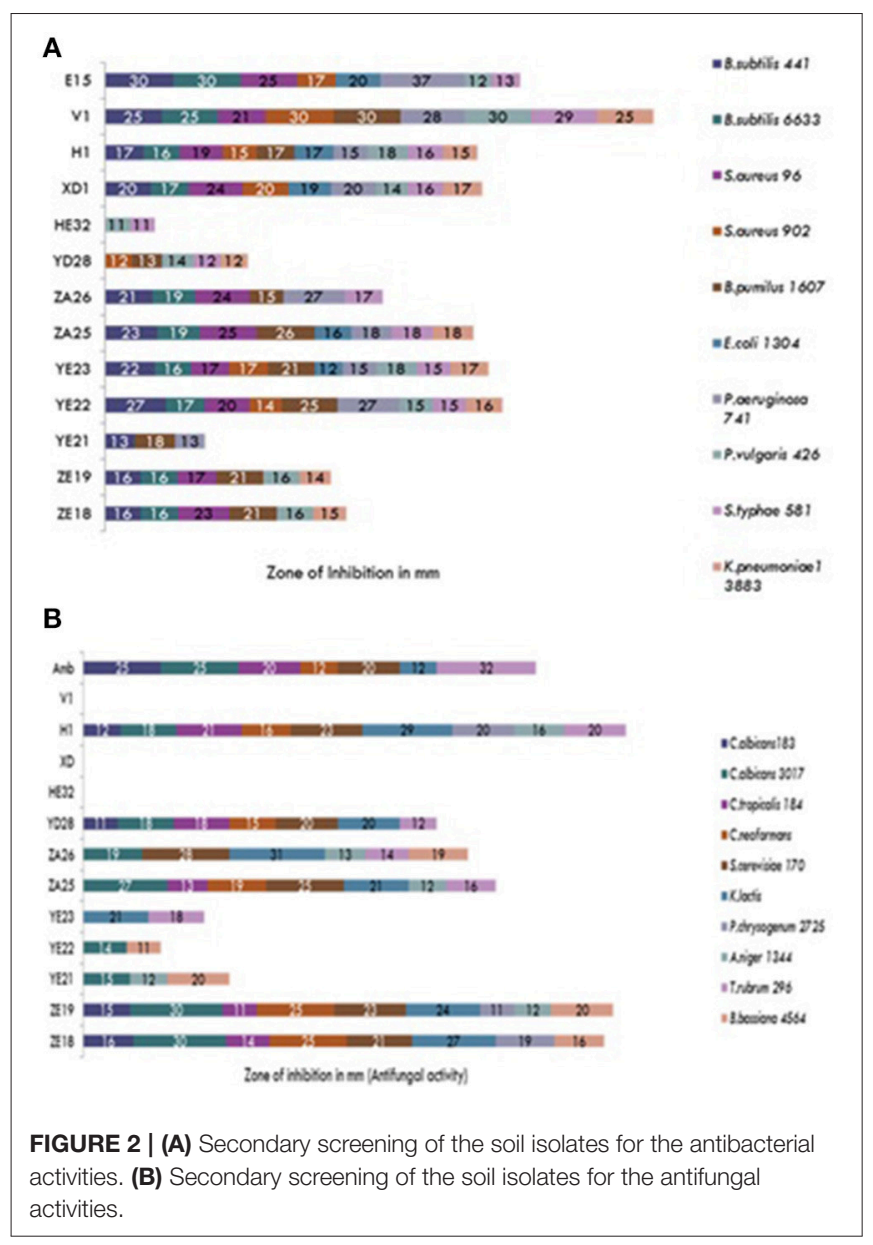

\section{Hierarchical Cluster Analysis}

Strains found to be active in primary and secondary screening were subjected to hierarchical cluster analysis based on 49 macroscopic, biochemical and physiological characters. The dendrograms based on the average linkage between the groups were generated by PASW Statistics (formerly SPSS Statistics) Version 18 software. Two broad clusters were generated (Figure 3). The first cluster contained four (ZE18, ZE19, ZA25, and ZA26) and the second cluster contained nine actinomycetes (YE23, RS25, H1, V1, N1, S1, YE22, YE21, and K1) isolates. The first cluster was further divided into two sub cluster sub-clad 1.1 and 1.2. The sub-clad 1.1 leaded ZA25 alone whereas sub-clad 1.2 further divided into 1.2.1 and 1.2.2 and so on, and ultimately formed a branched group of three isolates (ZE18, ZE19, and ZA26). Similarly, the second cluster was divided into sub-clad 2.1 and 2.2. The sub-clad 2.1 was further divided into 2.1.1 and 2.1.2 (YE21 and YE2,), whereas sub-clad 2.2 was further divided into 2.2.1 and 2.2.2 and so on, and ultimately formed a branched group of seven isolates (YE23, RS25, H1, V1, N1, S1, and K1).

Based upon the potential antimicrobial activities shown in the primary and secondary screenings, five out of thirteen strains were finally selected for further characterization using $16 \mathrm{~S}$ rRNA homology studies. The generated dendrogram showed that all the five strains (H1, V1, S1, RS25, and ZA25) were evolutionary far away from each other and displayed varying biochemical characteristics along with excellent antimicrobial activity. The diversity was maintained when we compared the antimicrobial activity data and the UV spectra of the metabolites. Strains, ZA25 and $\mathrm{H} 1$ belong to the main cluster I and II, respectively, found to produce both antibacterial and antifungal compounds having different UV absorbance. Similarly, H1, V1, and S1 produce antibacterial compounds and belong to the same cluster II; but their sub-clad were different. Variations in the UV absorbance of the metabolites supported the positional difference of the strains in the dendrogram. The 16S rRNA study for the strains H1, V1, and S1 were performed at Microbial Type Culture Collection (MTCC), Chandigarh, Punjab (India), and identified and submitted to MTCC as - Streptomyces xanthophaeus MTCC 11938, Streptomyces variabilis MTCC 12266, and Streptomyces xanthochromogenes MTCC 11937, respectively. Whereas, the $16 \mathrm{~S}$ rRNA homology sequence study for the strain RS25 and ZA25 was done at Genomebio Technologies Pvt. Ltd., Pune, Maharashtra (India) and identified and submitted to National Collection of Industrial Microorganisms (NCIM), CSIR-NCL, Pune, Maharashtra (India) as Streptomyces levis EU 124569 and Streptomyces sp. NCIM 5500, respectively.

\section{Partial Chemical Characterization of the Active Compounds}

The bioactive compounds were purified from the selected actinomycetes strains using silica column, with methanol:chloroform gradient as an eluting solvent system. The purified fractions were partially characterized by observing under UV absorbance $\left(\lambda_{\max }\right)$ and their absorption pattern to gather some preliminary information regarding the structure of the compound(s) (Table 4). Absorbance within UV range confirmed the presence of "unsaturation" in all the bioactive compounds. All the active metabolites except from the strain RS25 contained either carboxyl or peptide moiety in their structure. Absorbance band near $253 \mathrm{~nm}$ indicated the presence of benzene moiety in H1F2 (antifungal; AF), H1F1 (antibacterial; AB) and ZA25 AF strains. Among antifungal compounds, absence of conjugated absorption suggested non-polyene nature of H1F1 and ZA25 AF. The presence of carboxyl or peptide moieties was further confirmed by IR spectra of the compounds. Hydroxyl and conjugated carbonyl functional moiety in the compound were confirmed by the presence of absorption bands at 3425 and $1648 \mathrm{~cm}^{-1}$, respectively. The absorption bands detected at 3020 and $2927 \mathrm{~cm}^{-1}$ correspond to aromatic C-H and alkyl C-H stretching, respectively, and suggested the presence of aromatic ring in the antibacterial compound isolated from ZA25 and absence in the compound isolated from V1. Further, presence of aromatic ring was verified by the absorption bands at 1602 and $1504 \mathrm{~cm}^{-1}$ attributed to $\mathrm{C}=\mathrm{C}$ ring stretching.

\section{DISCUSSION}

During the screening of rare actinomycetes, fast growing bacterial colonies inhibits the colonization of actinomycetes on the isolation medium, hence in order to isolate actinomycetes, the growth of these bacteria should be inhibited. Pre-treatment of the soil samples reduced the growth of ubiquitous microbial species, 
TABLE 3 | Cultural and Biochemical characteristics of the producer strains.

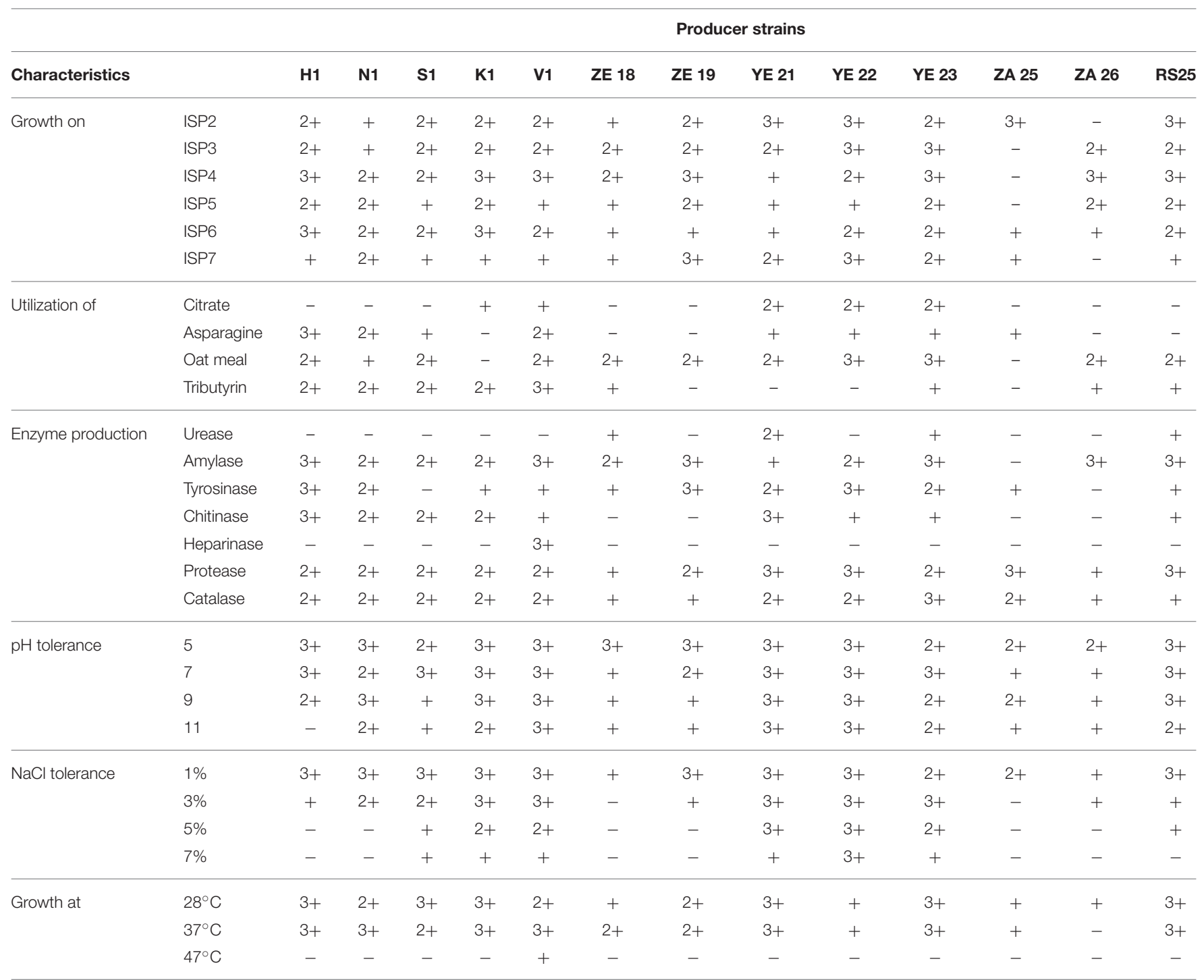

(-), No growth; (+1), Poor growth; (+2), Moderate growth; (+3), Heavy growth.

thereby facilitated the recovery of less-abundant microorganisms. The spores of actinomycetes and fungi generally resist desiccation and show slightly higher resistance toward wet or dry heat than other microbes (Hopwood and Wright, 1973). Pretreatment of the soil suspension with $1.5 \%$ phenol $\left(30^{\circ} \mathrm{C}\right.$ for $30 \mathrm{~min}$ ) lowered the number of bacteria, fungi, and other common actinomycetes by denaturing their proteins or by disrupting their cell membrane, however phenol-resistant actinomycetes were less affected during this process (Hayakawa et al., 1991). Earlier, Hayakawa et al. (1991) and Kim et al. (1995) reported the use of above mentioned pre-treatment of the soil samples for the isolation of actinomycetes, and they reported similar results, when the soil samples were pretreated.

A total of 15 isolated strains were subjected to antimicrobial screening and it was found that most of the isolates were active against gram positive bacteria. This was majorly attributed to the presence of lipopolysaccharide (LPS), a major structural unit of gram negative bacterial cell wall. LPS is hydrophobic in nature and makes the cell wall impermeable to lipophilic solutes, whereas in absence of LPS in the cell wall of gram positive bacteria, they become susceptible to the metabolites (Kim et al., 1994).

Out of 15 strains, thirteen showed strong antimicrobial activity and were selected for detailed microbial characterization studies. Melanin formation was also observed in almost all the selected strains, which is a main diagnostic feature of Streptomyces sp. (Singh et al., 2009). Further, five strains were characterized by using nucleotide sequencing and designated as H1: S. xanthophaeus MTCC 11938, V1: S. variabilis MTCC 12266, S1: S. xanthochromogenes MTCC 11937, RS25: S. levis EU124569, and ZA 25: Streptomyces sp. NCIM 5500. 
Evolutionary distance of the above five strains (H1, V1, S1, RS25, and ZA 25) was also analyzed by measuring their positions in the dendrogram. Based upon the diversity achieved by the morphological, biochemical, and the phylogenetic characteristics in the selected strains, we can speculate the possibility of involvement/role of diverse bioactive compounds from these strains, responsible for their broad-spectrum antimicrobial properties.

During the purification of the metabolites from ultra violet spectra, maximum absorbance between 205 and $216 \mathrm{~nm}$ suggested the presence of carboxyl or peptide moiety in the structure of all the active metabolites except from the strain RS25 (Singh et al., 2009; Kang et al., 2010). However, absorbance at 240-250 and at $332 \mathrm{~nm}$ predicted the presence of chromone like nucleus in strain RS25 (Griffiths and Ellis, 1972).

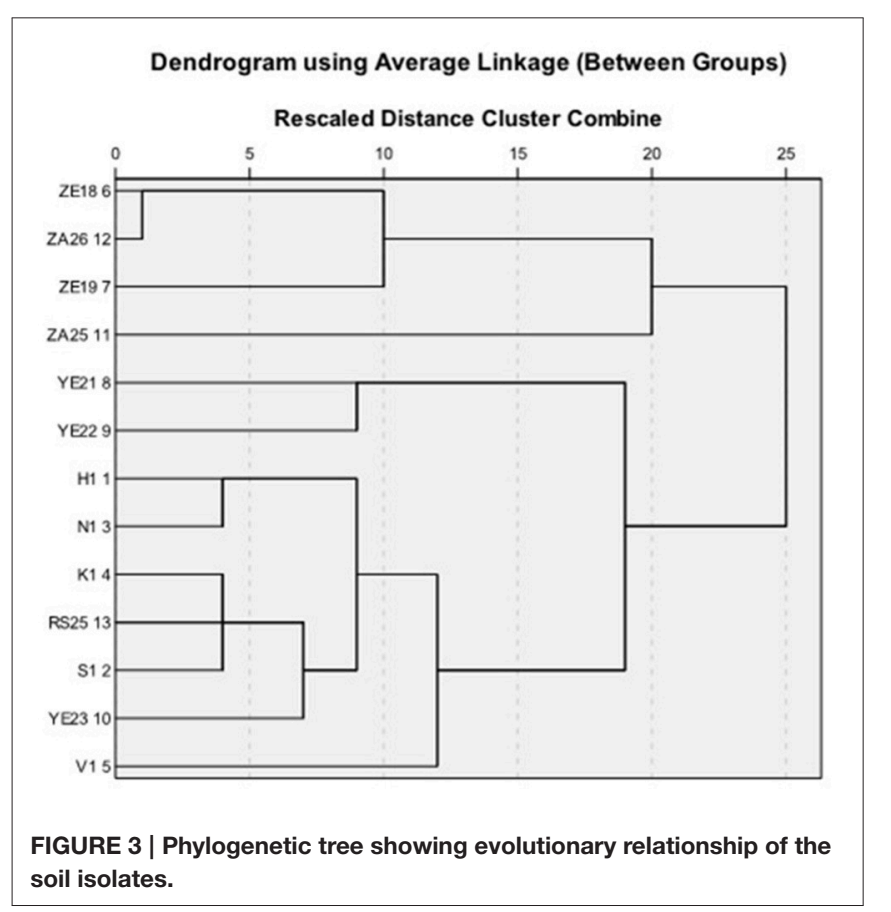

Pertinent literature about the producer strains were searched using SciFinder software program and it was found that $S$. xanthophaeus is known to produce diverse array of metabolites including Postproline endopeptidase, Benarthin, $\beta$-galactosidaseinhibiting isoflavonoids and geomycins (Brockmann and Musso, 1954; Hazato et al., 1979; Aoyagi et al., 1992; Shibamoto et al., 1993). S. xanthochromogenes are also known to produce diverse array of chemical compounds such as Diastereoisomeric I-Na, Nitropeptin, xanthicin (I), Pravastatin, reductiomycin, alkaloid AM-6201 (Arishima et al., 1956; Onda et al., 1982; Ohba et al., 1987; Otake et al., 1988; Cho et al., 1993; Zhang et al., 2008). Eleven compounds having same molecular formula have been reported from $S$. levis. Those were oleandomycin, 2-piperidinone and derivatives of either erythromycin or tylonolide. S. variabilis is reported for the production of L-Glutaminase, imunosuppresive, clavulanic acid, glycoside antibiotic and 1-hydroxy-1-norresistomycin (Abd-Alla et al., 2013, 2016; Marques et al., 2014; Ramalingam and Rajaram, 2016).

To the best of our knowledge, this is very first time we are reporting the antifungal activity of $S$. xanthophaeus and $S$. xanthochromogenes. Similarly, the compounds reported from $S$. levis didn't show the presence of chromone like structure. Also, the antifungal compound purified from S. xanthochromogenes was confirmed as chitinase by performing enzyme assay.

In conclusion, the results of morphological and biochemical characterization and the nature of compounds produced by the microbes established the diversity among the member of actinomycetes. The antimicrobial activities achieved in this study indicate that the isolated strains of Streptomyces sp. from different geographical niches of India have potential to produce diverse array of antimicrobial compounds that can be useful for many great applications and must be explored extensively. Currently, our research group and collaborators are actively involved in the chemical characterization of the active compounds identified in this study. In addition, in silico predictive studies for target prediction are under progress with our collaborator group. Hopefully, in our successive publication we would be reporting the complete information regarding the chemical characteristics of the

TABLE 4 | IR spectra of bioactive compounds isolated from the selected actinomycetes strains.

\begin{tabular}{|c|c|c|c|c|}
\hline Strains & Identification & Nature of purified compound & UV absorbance & IR frequency \\
\hline H1F1 & Streptomyces xanthophaeus & Antibacterial & 201,260 & $\begin{array}{l}3409,3019,2400,1658,1402,1384,1215,1053,948 \\
758,668\end{array}$ \\
\hline ZA25F1 & Streptomyces sp. & Antibacterial & 264,495 & $3411,3019,2400,1647,1403,1070,928,669$ \\
\hline V1 & Streptomyces variabilis & Antibacterial & 210 & $3412,2928,1646,1215,1070$ \\
\hline RS25 & Streptomyces levis & Antibacterial & $240-250$ and 332 & 3425 and 1648, 3020 and 2927, 1602 and $1504 \mathrm{~cm}^{-1}$ \\
\hline S1 & Streptomyces xanthochromogenes & Antifungal & 205,490 & $3411,3020,1646,1403,1385,1216,1069,769$ \\
\hline $\mathrm{H} 1 \mathrm{~F} 2$ & Streptomyces xanthophaeus & Antifungal & 201,265 & $\begin{array}{l}3850,3431,3020,2399,2110,1637,1403,1385 \\
1017,669\end{array}$ \\
\hline ZA25F2 & Streptomyces sp. & Antifungal & $205,248,263$ & $\begin{array}{l}3850,3366,2945,2833,2518,2042,1638,1449 \\
1218,1113,757,666\end{array}$ \\
\hline
\end{tabular}


"actives" identified in this study and their targets predicted through in silico binding studies and their experimental validation.

\section{AUTHOR CONTRIBUTIONS}

Conceived and designed the study and experiments: VS, $\mathrm{SH}, \mathrm{HS}, \mathrm{JV}, \mathrm{KV}, \mathrm{RS}, \mathrm{AJ}$, CT. Performed the experiments: VS, HS, JV, KV, RS. Analyzed the data: VS, SH, AJ. Contributed reagents/materials/analysis tools: VS, SH, AJ, CT. Wrote the paper: VS, SH, AJ, CT. All authors reviewed the manuscript.

\section{REFERENCES}

Abd-Alla, M. H., El-Sayed, E. S. A., and Rasmey, A. H. M. (2013). Biosynthesis of L-glutaminase by Streptomyces variabilis ASU319 isolated from rhizosphere of triticum vulgaris. Univ. J. Microbiol. Res. 1, 27-35. doi: 10.13189/ujmr.2013.010301

Abd-Alla, M. H., Rasmey, A. H. M., El-Sayed, E. S. A., El-Kady, I. A., and Yassin, I. M. (2016). Biosynthesis of anti-inflammatory immunosuppressive metabolite by Streptomyces variabilis ASU319. Eur. J. Biol. Res. 6, 152-169. Available online at: http://www.journals.tmkarpinski.com/index.php/ejbr/article/view/455

Aoyagi, T., Hatsu, M., Kojima, F., Hayashi, C., Hamada, M., and Takeuchi, T. (1992). Benarthin: a new inhibitor of pyroglutamyl peptidase. i taxonomy, fermentation isolation and biological activities. J. Antibiot. 45, 1079-1083. doi: 10.7164/antibiotics.45.1079

Arishima, M., Sakamoto, J., and Sato, T. (1956). An antibiotic Streptomyces no. 689 strain. I. Taxonomic studies. Nippon Nogei Kagaku Kaishi 30, 469-471. doi: 10.1271/nogeikagaku1924.30.8_469

Berdy, J. (2005). Bioactive microbial metabolites, a personal view. J. Antibiot. 58, 1-26. doi: 10.1038/ja.2005.1

Brockmann, H., and Musso, H. (1954). Antibiotics from actinomycetes. XXIX. Geomycin 2. Chem. Ber. 87, 1779-1799. doi: 10.1002/cber.19540871128

Cho, H., Beale, J. M., Graff, C., Mocek, U., Nakagawa, A., Omura, S., et al. (1993). Studies on the biosynthesis of the antibiotic reductiomycin in Streptomyces xanthochromogenus. J. Am. Chem. Soc. 115, 12296-12304. doi: 10.1021/ja00079a009

Griffiths, P. J. F., and Ellis, G. P. (1972). Benzopyrones-VI1: the ultraviolet absorption spectra of chromone and 2-substituted chromones. Spectrochim. Acta A Mol. Spectros. 28, 707-713. doi: 10.1016/0584-8539(72)80039-4

Hayakawa, M. (2008). Studies on the isolation and distribution of rare actinomycetes in soil. Actinomycetologica 22, 12-19. doi: 10.3209/saj.SAJ220103

Hayakawa, M., Sadakata, T., Kajiura, T., and Nonomura, H. (1991). New methods for the highly selective isolation of Micromonospora and Microbispora from soil. J. Fermen. Bioeng. 72, 320-326. doi: 10.1016/0922-338X(91)90080-Z

Hazato, T., Naganawa, H., Kumagai, M., Aoyagi, T., and Umezawa, H. (1979). Beta-Galactosidase-inhibiting new isoflavonoids produced by actinomycetes. J. Antibiot. 32, 217-222. doi: 10.7164/antibiotics.32.217

Holt, J. G., Krieg, N. R., Sneath, P. H. A., Staley, J. T., and Williams, S. T. (1994). Bergey's Manual of Determinative Bacteriology, 9th Edn. Baltimore, MD; Philadelphia; Hong Kong; London; Munch; Sydney; Tokyo: William and Wilkins.

Hopwood, D. A., and Wright, H. M. (1973). A plasmid of Streptomyces coelicolor carrying a chromosomal locus and its inter-specific transfer. J. Gen. Microbiol. 79, 331-342. doi: 10.1099/00221287-79-2-331

Kang, M. J., Strap, J. L., and Crawford, D. L. (2010). Isolation and characterization of potent antifungal strains of the Streptomyces violaceusniger clade active against Candida albicans. J. Ind. Microbiol. Biotechnol. 37, 35-41. doi: 10.1007/s10295-009-0641-9

Kekuda, P. T. R., Onkarappa, R., and Jayanna, N. D. (2014). Characterization and antibacterial activity of a glycoside antibiotic from Streptomyces

\section{FUNDING}

The financial support for this study was available from the Department of Science and Technology, Ministry of Science and Technology, Government of India, New Delhi, under Fast Track Fellowship awarded to VS (Grant No. SR/FT/LS190/2009).

\section{ACKNOWLEDGMENTS}

The authors are grateful to acknowledge the laboratory facility provided by the CSIR-CDRI, Lucknow, UP, India for this study.

variabilis PO-178. Sci. Technol. Arts Res. J. 3, 116-121. doi: 10.4314/star v3i4.17

Kim, C., Lee, K., Kwon, O., Park, D., and Shimazu, A. (1995). Isolation of rare actinomycetes on various types of soil. Kor. J. Appl. Microbiol. Biotechnol. 23, $36-42$.

Kim, C., Lee, K., Kwon, O., Yoo, I., and Shimazu, A. (1994). Selective isolation of actinomycetes by physical pretreatment of soil sample. Kor. J. Appl. Microbiol. Biotechnol. 22, 222-225.

Kurtböke, D. I., Chen, C. F., and Williams, S. T. (1992). Use of polyvalent phage for reduction of streptomycetes on soil dilution plates. J. Appl. Bacteriol. 72, 103-111. doi: 10.1111/j.1365-2672.1992.tb01810.x

Madigan, M. T., Martiko, J. M., and Parker, J. (1997). "Antibiotics: isolation and characterization," in Brock Biology of Microorganisms, 8th Edn., ed M. T. Madigan (New Jersey: Prentice-Hall International Inc.), 440-442.

Marques, D. A. V., Santos-Ebinuma, V. D. C., de Oliveira, P. M. S., de Souza Lima, G. M., Araújo, J. M., Lima-Filho, J. L., et al. (2014). Screening of wild type Streptomyces isolates able to overproduce clavulanic acid. Braz. J. Microbiol. 45, 919-928. doi: 10.1590/S1517-83822014000300022

Ohba, K., Nakayama, H., Furihata, K., Shimazu, A., Endo, T., Seto, H., et al. (1987). Nitropeptin, a new dipeptide antibiotic possessing a nitro group. J. Antibiot. 40, 709-713.

Okami, Y., and Hotta, K. (1988). "Search and discovery of new antibiotics," in Actinomycetes in Biotechnology, eds M. Goodfellow, S. T. Williams, and M. Mordarski (London: Academic Press), 37-67.

Onda, M., Konda, Y., Hinotozawa, K., and Omura, S. (1982). The alkaloid AM6201 from Streptomyces xanthochromogenus. Chem. Pharmaceut. Bull. 30, 1210-1214. doi: $10.1248 / \mathrm{cpb} .30 .1210$

Otake, N., Seto, H., Nakayama, H., Endo, T., Oba, K., Iwata, M., et al. (1988). Antibiotic 6257 manufacture with Streptomyces for Pyricularia oryzae infection control in rice. Jpn. Kokai Tokkyo Koho. JP 63126495 (Accessed May 30, 1988).

Praveen, V., Tripathi, D., Tripathi, C. K. M., and Bihari, V. (2008). Nutritional regulation of actinomycin-D production by a new isolate of Streptomyces sindenensis using statistical methods. Indian J. Exp. Biol. 46, 138-144.

Ramalingam, V., and Rajaram, R. (2016). Antioxidant activity of 1-hydroxy-1norresistomycin derived from Streptomyces variabilis KP149559 and evaluation of its toxicity against zebra fish Danio rerio. RSC Adv. 6, 16615-16623. doi: 10.1039/C5RA22558B

Shibamoto, N., Terasawa, T., Okamoto, R., Shin, T., and Murao, S. (1993). Novel postproline endopeptidase and its manufacture with Streptomyces species. Jpn. Kokai Tokkyo Koho. Patent No. JP 05244947, Indexing: Fermentation and Bioindustrial Chemistry (Section 16-4).

Shirling, E. B., and Gottlieb, D. (1966). Methods for characterization of Streptomyces sp. Int. J. Syst. Bacteriol. 16, 313-340. doi: 10.1099/00207713-16-3-313

Singh, V., and Tripathi, C. K. M. (2011). Olivanic acid production in fed batch cultivation by Streptomyces olivaceus MTCC 6820. Res. J. Pharmaceut. Biol. Chem. Sci. 2, 726-731.

Singh, V., Praveen, V., Banga, J., and Tripathi, C. K. M. (2009). Antimicrobial activities of microbial strains isolated from soil of stressed ecological 
niches of Eastern Uttar Pradesh, India. Indian J. Exp. Biol. 47, 298-303.

Takahashi, Y., and Omura, S. (2003). Isolation of new actinomycete strains for the screening of new bioactive compounds. J. Gen. Appl. Microbiol. 49, 141-154. doi: 10.2323 /jgam.49.141

Tanaka, Y., and Omura, S. (1990). Metabolism and products of actinomycetes. An introduction. Actinomycetolgica 4, 13-14. doi: 10.3209/saj.4_13

Williams, S. T., Goodfellow, M., and Alderson, G. (1989). "Bergey's manual of systematic bacteriology," in Genus Streptomyces Waksman and Henrici 1943, 339AL, Vol. 4, eds S. T. Williams, M. E. Sharpe, and J. G. Holt (Baltimore, MD: Williams and Wilkins), 2452-2492

Wu, R. Y. (1984). Studies on the Streptomyces SC4. II Taxonomic and biological characteristics of Streptomyces strain SC4. Bot. Bull. Acad. Sin. 25, 111-123.

Zhang, L., Zhang, J., Yang, W., and Bai, G. (2008). Classification of Streptomyces strain Z314 and purification of its product pravastatin. Wei Sheng Wu Xue Bao $48,33-37$.
Conflict of Interest Statement: The authors declare that the research was conducted in the absence of any commercial or financial relationships that could be construed as a potential conflict of interest.

The reviewer PCV declared a shared affiliation, though no other collaboration, with the authors to the handling Editor, who ensured that the process nevertheless met the standards of a fair and objective review.

Copyright (c) 2016 Singh, Haque, Singh, Verma, Vibha, Singh, Jawed and Tripathi. This is an open-access article distributed under the terms of the Creative Commons Attribution License (CC BY). The use, distribution or reproduction in other forums is permitted, provided the original author(s) or licensor are credited and that the original publication in this journal is cited, in accordance with accepted academic practice. No use, distribution or reproduction is permitted which does not comply with these terms. 